\title{
Monocular 3D Object Detection with Decoupled Structured Polygon Estimation and Height-Guided Depth Estimation
}

\author{
Yingjie Cai, ${ }^{1}$ Buyu Li, ${ }^{1}$ Zeyu Jiao, ${ }^{2}$ Hongsheng Li, ${ }^{1}$ Xingyu Zeng, ${ }^{2}$ Xiaogang Wang ${ }^{1}$ \\ ${ }^{1}$ The Chinese University of Hong Kong, ${ }^{2}$ Sensetime Group Limited \\ caiyingjie@link.cuhk.edu.hk, \{jiaozeyu, zengxingyu\}@ sensetime.com, \{byli, hsli, xgwang\}@ee.cuhk.edu.hk
}

\begin{abstract}
Monocular 3D object detection task aims to predict the 3D bounding boxes of objects based on monocular RGB images. Since the location recovery in $3 \mathrm{D}$ space is quite difficult on account of absence of depth information, this paper proposes a novel unified framework which decomposes the detection problem into a structured polygon prediction task and a depth recovery task. Different from the widely studied 2D bounding boxes, the proposed novel structured polygon in the $2 \mathrm{D}$ image consists of several projected surfaces of the target object. Compared to the widely-used 3D bounding box proposals, it is shown to be a better representation for 3D detection. In order to inversely project the predicted 2D structured polygon to a cuboid in the 3D physical world, the following depth recovery task uses the object height prior to complete the inverse projection transformation with the given camera projection matrix. Moreover, a fine-grained 3D box refinement scheme is proposed to further rectify the $3 \mathrm{D}$ detection results. Experiments are conducted on the challenging KITTI benchmark, in which our method achieves state-of-the-art detection accuracy.
\end{abstract}

\section{Introduction}

3D object detection is an important computer vision task since it is an essential component of autonomous driving and robot perception to avoid collisions with surrounding objects. Most existing 3D object detection methods heavily rely on LiDAR devices to obtain accurate and direct depth measurements. However, such sensors can not widely adopted due to the expensive cost and limited perception range $(\sim 100 \mathrm{~m})$. The farther away the objects are, the fewer and sparser depth measurements would be on the objects. In contrast, cameras are much cheaper and can be installed on any vehicles. This paper mainly focuses on 3D detection with monocular images. In general, a 3D bounding box can be described by 7 parameters in autonomous driving scenarios, i.e. the location $(x, y, z)$, size $(l, w, h)$ and orientation $\theta$ on the ground. For 3D detection from monocular images, recovering the location in $3 \mathrm{D}$ space is challenging on account of the absence of the accurate depth measurements. As illustrated in Fig. 1, given an accurate 2D bounding box of an

Copyright (C) 2020, Association for the Advancement of Artificial Intelligence (www.aaai.org). All rights reserved.

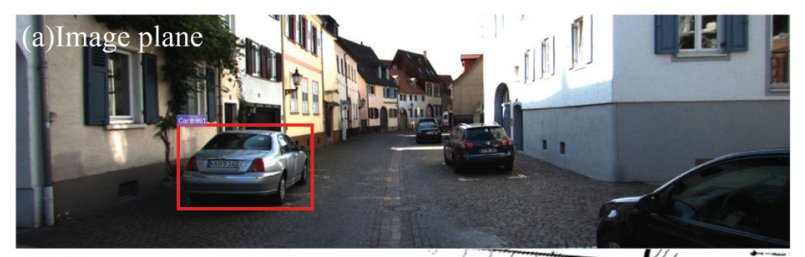

(b)3D space

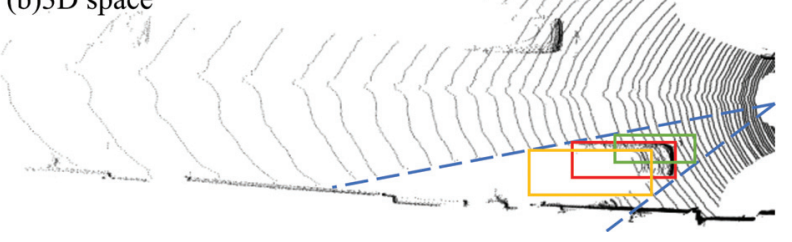

Figure 1: 3D detection from 2D monocular images is challenging as even accurate 2D detection boxes (top) correspond to ambiguous 3D detection boxes (bottom). Best viewed in color.

object (Fig. 1 (a)), its 3D location is still difficult to recover because one $2 \mathrm{D}$ box has an infinite number of corresponding 3D boxes (Fig. 1 (b)) according to 2D-to-3D projection. However, the projection of the $3 \mathrm{D}$ bounding box on the $2 \mathrm{D}$ image plane is unique and much easier to estimate with features in a $2 \mathrm{D}$ image. Since the projected 3D box follows prior knowledge of being a polygon consisting of several quadrilaterals (corresponding to the visible surfaces of the 3D box), we refer it as structured polygon for convenience. The 3D box can be completely recovered given the structured polygon, depth and the projection matrix. As the camera projection matrix is generally known in auto-driving scenarios, the only additional information required is the depth of the object.

Inspired by the analysis above, we propose a novel framework that decomposes the 3D object detection task into a structured polygon prediction task and a depth estimation task. Different from the commonly used 2D bounding boxes in most previous works (Chen et al. 2016), (Mousavian et al. 2017), (Xu and Chen 2018) and (Qi et al. 2018), the structured polygon can provide richer information for the $3 \mathrm{D}$ box recovery. Since the challenging depth estimation task is decoupled, the specific module can be designed to accurate 


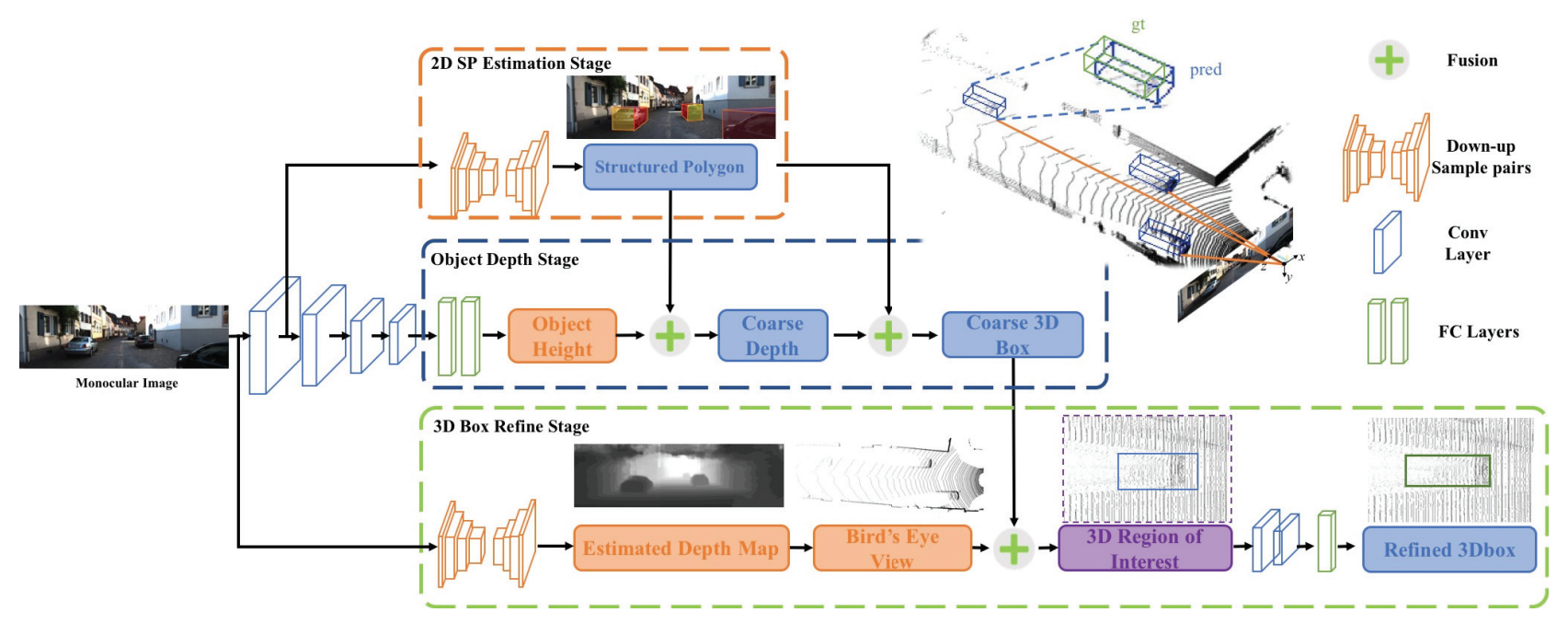

Figure 2: The overall framework (Decoupled-3D) decouples the monocular 3D object detection problem into sub-tasks. The overall network consists of three parts. (Top row) The 2D structured polygons are generated with a stacked hourglass network. (Middle row) Object depth stage utilizes 3D object height as a prior to recover the missing depth of the object. (Bottom row) $3 \mathrm{D}$ box refine stage rectifies coarse 3D boxes using bird's eye view features in $3 D-R O I$ s. Best viewed in color.

tackle it. Moreover, the prediction of other parameters, e.g., the orientation of the object and the aspect ratio, would not be affected by depth estimation. Therefore, our framework has significant superiority over existing works that estimate all the 3D parameters simultaneously (Chen et al. 2018), (Chen et al. 2017), (Xu and Chen 2018), (Qi et al. 2018).

To obtain the structured polygon, we regress the projection points of the eight vertices of the cuboid via a stacked hourglass network. With the predicted structured polygon, we propose an efficient method to estimate the depth. Specifically, we use the object height as a prior to compute the inverse projection transformation from the $2 \mathrm{D}$ image plane to $3 \mathrm{D}$ space with the given camera projection matrix. With the estimated depth and the obtained structured polygon, we can consequently recover the complete $3 \mathrm{D}$ box.

The 3D box obtained from previous steps is actually a coarse estimation. We further propose a 3D box refinement scheme to rectify the coarse 3D boxes of the objects. We use the local features around and within the coarse 3D box for the refinement. The features are extracted from the bird's eye view map of the estimated depth map by a monocular depth estimation algorithm DORN (Fu et al. 2018). In contrast to previous works that refine the $3 \mathrm{D}$ boxes with $2 \mathrm{D}$ imagelevel features such as MGR (Qin, Wang, and Lu 2019) and MLF (Xu and Chen 2018), we refine the deviations of coarse boxes with bird's eye view map containing direct spatial information. The ingenious design can align the coarse boxes adaptively and substaintially enhances the accuracy of 3D boxes.

To validate the effectiveness of our method, we perform thorough experiments on the challenging 3D object detection benchmark KITTI (Geiger, Lenz, and Urtasun 2012) and achieve new state-of-the-art performance under both $A P_{B E V}$ and $A P_{3 D}$ metrics. The contributions are summarized as following three-fold:
- A novel framework, which decomposes the challenging 3D detection problem into sub-tasks of image based structured polygon prediction and object depth estimation, is proposed. The two decomposed sub-tasks can be better tackled.

- An efficient object depth estimation approach is proposed, which uses the object height as a prior. Combined the depth with the structured polygon, coarse 3D boxes can be obtained.

- A fine-grained 3D box refinement scheme is proposed. Different from the existing methods, we rectify the coarse boxes with bird's eye view map, which significantly improves the accuracy of the $3 \mathrm{D}$ boxes.

\section{Related work}

We briefly review recent works based on LiDAR data, stereo images and monocular images.

LiDAR-based 3D Object Detection. Most state-of-the-art 3D object detection methods reconstruct 3D bounding box using point clouds from LiDAR. (Luo, Yang, and Urtasun 2018) and (Zhou and Tuzel 2018) quantize the raw point cloud by using voxel grid and then feed the structured voxel grid to 2D or 3D CNN to detect 3D objects. (Qi et al. 2018) and (Shi, Wang, and Li 2019) directly exploit raw point cloud to generate $3 \mathrm{D}$ bounding boxes instead of quantizing to voxel grid with less information lossing. They respectively uses 2D bounding box and segmentation to lock effective point cloud and both encode point cloud via PointNet++ (Qi et al. 2017). Our method focuses on monocular data setting and unavoidably suffers from the lack of accurate and direct depth measurements.

Stereo-based 3D Object Detection. There are several 
works are based on stereo vision. Stereo R-CNN (Li, Chen, and Shen 2019) utilizes stereo RPN to detect 3D objects on left and right images simultaneously and tries novel pixel-level refinement based on stereo matching to refine 3D boxes. 3DOP (Chen et al. 2015) assumes enormous $3 \mathrm{D}$ candidates and exploits ground-plane prior and object size to set up a energy function to filter the candidates. Stereo images provide more information than monoculars. However, stereo setting has high requirements during the camera installation while our approach only needs a single image, which can be more flexible in real cases.

Monocular-based 3D Object Detection. More and more recent works are based on monocular images even through it is the most difficult. MGR (Qin, Wang, and Lu 2019) and Mono3D (Chen et al. 2016) encode RGB image feature to $2 \mathrm{D} \mathrm{CNN}$ to regress $3 \mathrm{D}$ proposals and further refine the proposals with superimposed 2D features. Mono3D generates a diverse set of 3D candidate boxes first and exploites ground plane prior and 2D cues including segmentation and object size to filter the candidates. Pseudo-LiDAR (Wang et al. 2019) transforms the depth map into pseudo point clouds and feeds the points into LiDAR based methods. (Kehl et al. 2017), (Pepik et al. 2015) and (Chabot et al. 2017) adopt CAD models to build templates for better supervision. Deep3Dbox (Mousavian et al. 2017) leverages the geometry constrains between $3 \mathrm{D}$ and $2 \mathrm{D}$ bounding box to recover the $3 \mathrm{D}$ poses. These methods use more information from the superimposed 2D image level features or additional CAD models to constrain. However, our method decomposes the problem in a novel approach and designs specific strategies for each subtasks to achieve better 3D object detection.

\section{Our Approach}

In this section, we present our proposed framework for 3D object detecting from monocular images. First, we introduce the overall formulation of our architecture. We then introduce 3D coarse box estimation with structured polygon and depth estimation. Finally, a 3D box refinement scheme to rectify coarse boxes is demonstrated. We name our 3D object detection method via decoupled tasks as Decoupled-3D, as illustrated in Fig. 2.

\section{Decoupled Tasks}

As introduced, since the estimation of depth is the most strenuous part for monocular based 3D object detection, we decouple the depth from complicated 3D box estimation and decompose the task into structured polygon prediction and coarse-to-fine depth estimation sub-tasks.

In $3 \mathrm{D}$ object detection, each object can be covered by a minimal cuboid in the 3D space, denoted by $B_{3 D}$. A cuboid contains eight vertices $P_{i}=\left[X_{i}, Y_{i}, Z_{i}\right]^{T} \in \mathbb{R}^{3}, i=1, \cdots, 8$, as the left blue cuboid shown in Fig. 3. An object corresponds to a special structured polygon on the 2D image plane via 3D-to-2D projection. A structured polygon contains eight projected vertices $\left\{p_{i}=\left[u_{i}, v_{i}\right]^{T} \mid i=1, \cdots, 8\right\}$ as the 2D vertices shown in Fig. 3 (right). Given the camera

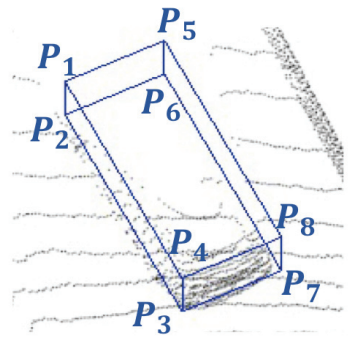

$3 D$ bounding box

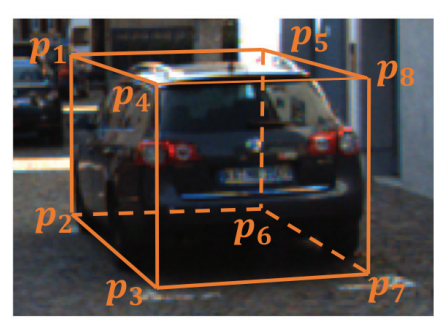

Structured polygon
Figure 3: 3D bounding box (left) and structured polygon (right). Best viewed in color.

intrinsic matrix $K$, the projection of a 3D vertex $P_{i}$ on the image plane is formulated as the following equation:

$$
K \cdot\left[X_{i}, Y_{i}, Z_{i}\right]^{\mathrm{T}}=\left[u_{i}, v_{i}, 1\right]^{\mathrm{T}} \cdot Z_{i},
$$

where $Z_{i}$ is the depth of the vertex $P_{i}$. Given $p_{i}$ and $Z_{i}, P_{i}$ can be obtained as:

$$
\left[X_{i}, Y_{i}, Z_{i}\right]^{\mathrm{T}}=K^{-1} \cdot\left[u_{i}, v_{i}, 1\right]^{\mathrm{T}} \cdot Z_{i} .
$$

According to this equation, to estimate $B_{3 D}$, we just need $K$, projected 2D vertices $p_{i}$ and the corresponding depth $Z_{i}$. As the camera intrinsic matrix $K$ is generally known, the remaining problem is the estimation of the $2 \mathrm{D}$ vertices of the structured polygon and their corresponding depths.

\section{D Structured Polygon Estimation}

In order to first obtain the locations of objects in the 2D image, we predict the 2D bounding box of each object by Faster RCNN (Ren et al. 2015).

For structured polygon estimation, we propose to regress the 2D image coordinates of the 8 vertices based on the features extracted from the object area. However, it is still difficult to find accurate position in occlusion areas, textureless regions and reflective surfaces. As shown in Fig. 4, the vertices are projected on texture-less background such as ground plane and wall without strong physical meaning. Solely applying the local feature is generally insufficient for accurate estimation in such challenging regions. Therefore, global context information should be incorporated to infer accurate positions of the vertices.

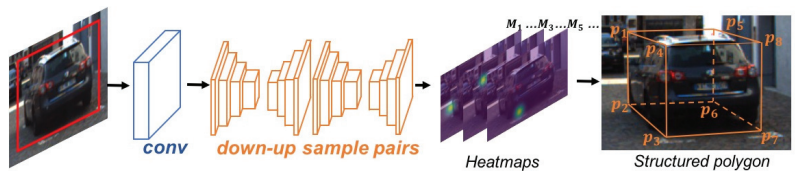

Figure 4: Structured polygon estimation aims to estimate the 2D locations of the projected vertices.

To capture more global context information, we adopt a stacked hourglass architecture after several shallow convolution layers (res2 in ResNet). This architecture consists of two repeated top-down/bottom-up hourglass modules (Newell, Yang, and Deng 2016). The fusion of features from multi-scales can integrate both local and global 
features to obtain accurate vertices positions. Each of the eight projected vertices has a corresponding output heatmap from the network, as shown in Fig. 4. We use $M_{i}$ to denote the heatmap of the projected vertex $p_{i}$, and $M_{i}(u, v)$ is the value of the pixel $(u, v)$ on the heatmap, which represents the probability of the vertex locating at this location. The supervision of each vertex is a label map $\hat{M}_{i}$ with the ground truth position being one and others being zeros. We use the Euclidean loss function for training, which can help the model converge faster:

$$
L_{s p}=\sum_{i}\left\|M_{i}-\hat{M}_{i}\right\|_{2}^{2}
$$

During testing, the vertex position is estimated as the location with the highest probability.

$$
\hat{p}_{i}=\underset{(u, v)}{\arg \max } M_{i}(u, v)
$$

The label of the eight projected vertices can be obtained via 3D-to-2D projection with Eq. (1) from 3D coordinates of the vertices.

\section{Height-Guided Depth Estimation}

The depth of an object is the most challenging parameter to estimate due to the fact that this information is missing after 3D-to-2D projection. Therefore, instead of directly regressing the strenuous depth from image-level features, we choose to recover the depth via camera projection principle. Based on the projection principle, we adopt a simple, but effective strategy for the missing depth via structured polygon and a 3D physical prior, object height.

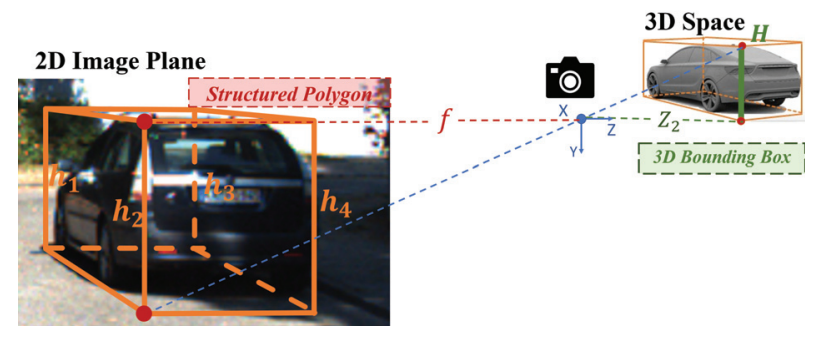

Figure 5: Height-Guided Depth Estimation. Combine object height $H$ and corresponding pixel value $h$ to estimate object depth. Best viewed in color.

As shown in Fig. 5, $f$ is the camera focal length, $H$ represents the $3 \mathrm{D}$ height of object and $h_{j}$ for $j=1,2,3,4$ is the projected height of one vertical edge of the cuboid. The height values of the four vertical edges in 3D space are the same, while the projected height of the four vertical edges are different due to their different locations in the 3D space. Fig. 5 clearly shows the 3D-to-2D projection process of one vertical edge (i.e., $h_{2}$ ). Therefore, the corresponding depth $\left(Z_{j}\right)$ of each vertical edge of the cuboid can be expressed as

$$
Z_{j}=f \cdot H / h_{j} \quad \text { for } j=1,2,3,4
$$

where $h_{j}$ can be directly obtained from the estimated structured polygon, which is the pixel distance of two projected vertices. For object height $H$, which represents real height in 3D space, an intuitive idea is to use the average value $A_{H}$ obtained from the statistics of the height values in data set. However, the average height is not accurate enough for each instance. So we estimate the height of each object. Specifically, we pool the RoI feature of an object on the feature map from Res4, and then use 2 fully connected layers to predict the height. Instead of regressing the ground truth height $G_{H}$ directly, our regression target $t_{H}$ is the scale change:

$$
t_{H}=\log \left(G_{H} / A_{H}\right)
$$

The Smooth- $l_{1}$ (Girshick 2015) loss function is adopted for the training of the regressor, since Smooth- $l_{1}$ is less sensitive to outliers. Further, according to Eq. (2), the coordinates of eight vertices in 3D space can be obtained via generated projected vertices and depths.

With the eight 3D vertices, we use an average operation to obtain a coarse 3D box. Specifically, in KITTI dataset the location of an object is defined as the position of the bottom center of its 3D bounding box, so we use the average of the midpoints of the diagnal $P_{2} P_{7}$ and $P_{3} P_{6}$ to estimate the location $(x, y, z)$. The $l$ can be calculated using the average of distances of $P_{2} P_{3}, P_{6} P_{7}, P_{1} P_{4}$ and $P_{5} P_{8} . h$ and $w$ are calculated using the similar way. Orientation $\theta$ comes from the average of four vertors $\overrightarrow{P_{3} P_{2}}, \overrightarrow{P_{7} P_{6}}, \overrightarrow{P_{4} P_{1}}$ and $\overrightarrow{P_{8} P_{5}}$.

\section{D Box Refinement}

The 3D box obtained from previous steps is actually a coarse estimation. But the error is usually minor and the groundtruth is just located nearby. As shown in bird's eye view of Fig. 6 (left), the predictions have deviations about $1 \mathrm{~m}$.

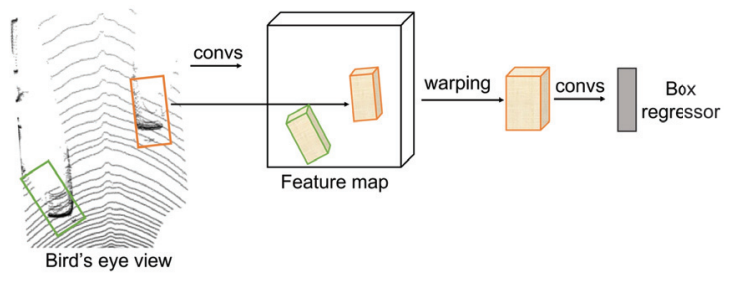

Figure 6: 3D Box Refinement. Rectify coarse boxes with bird's eye view map.

Based on this fact, the proposed method tailors a finegrained refinement scheme for 3D detection. Different from existing methods exploiting image-level features or frontview depth map, we leverage bird's eye view map, which contains direct spatial information, to rectify coarse boxes. The well-designed scheme can adjust coarse $3 \mathrm{D}$ boxes to better locations in nearby region. For convenience, we refer this Fine-Grained refinement strategy as $F G$.

Bird's eye view maps are transformed from predicted monocular depth maps using DORN (Fu et al. 2018). The details for the transformation process are outlined in Implementation Details. We take an entire bird's eye view map and a set of coarse boxes as input. The whole map is processed by a CNN to produce a feature map. Then, for each object we adopt a warping operation of the region on the 
feature map to extract a fixed-length feature vector. The region has $2 \mathrm{x}$ size of the coarse box to ensure that targets are within this region. Since this region contains direct 3D information, we named it $3 D-R O I$ for convenience. Each feature vector is fed into a sequence of convolution layers followed by a fully-connected layer that outputs residual values $(\delta x, \delta y, \delta z, \delta l, \delta w, \delta h, \delta \theta)$ based on the coarse 3D box. A Smooth- $l_{1}$ loss is used for the training of this network.

In this way, the deviations of coarse boxes are removed, which surges the performance. We argue that bird's eye view map with direct 3D spatial information is much more suitable for fine-grained refinement in 3D task than 2D imagelevel features or front-view depth map. Detailed analysis and comparisons are in Ablation Study.

\section{Experiments}

We evaluate the proposed method on the KITTI object detection benchmark (Geiger, Lenz, and Urtasun 2012) and split training images into training set and validation set following the commnly used train/val split mentioned in 3DOP (Chen et al. 2018). In the dataset, objects are divided into three difficulty regimes: easy, moderate and hard, according to the 2D bounding box height, occlusion and truncation degrees following the KITTI official standard. For all experiments, we follow most previous methods to focus on vehicle category as it has the majority of samples in the dataset.

\section{Implementation Details}

ResNet-50 (He et al. 2015) is selected as our basic backbone to extract features, which is initialized by pre-trained weights on ImageNet (Russakovsky et al. 2015). The initial learning rate is 0.001 for the previous $30 \mathrm{~K}$ interactions and then 0.0001 for another $10 \mathrm{~K}$ iterations. For the $3 \mathrm{D}$ box refinement, we consider the following range:

$-25 m \leq X \leq 25 m,-1.5 m \leq Y \leq 4.09 m, 0 \leq Z \leq 50 m$

The predicted depth map is from a monocular based method DORN (Fu et al. 2018). The row and column represent leftright (i.e., $X$ ) direction and the depth (i.e., $Z$ ) direction respectively, and the top-down value, $Y$ is mapped to a slice at each $(X, Y)$ location on bird's eye view maps. The width and height of $3 D-R O I$ are set as 256 and 456 respectively. The size is computed from the statistics to ensure targets are within this region. For the object height, we use $1.46 \mathrm{~m}$ as the average value.

\section{Comparison with Other Methods}

We compare with state-of-the-art monocular based methods including Mono3D (Chen et al. 2016), Deep3Dbox (Mousavian et al. 2017), MLF (Xu and Chen 2018), ROI-10D (Manhardt, Kehl, and Gaidon 2019), GS3D (Li et al. 2019), MGR (Qin, Wang, and Lu 2019), MonoPSR (Ku, Pon, and Waslander 2019) and Pseudo-LiDAR (Wang et al. 2019).

Metrics. The proposed method is evaluated by Average Precision on both bird's eye view $\left(A P_{B E V}\right)$ and $3 \mathrm{D}$ detection $\left(A P_{3 D}\right)$ metrics. $A P_{B E V}$ evaluates whether the prediction is accurate by calculating the intersection over union (IoU) with ground-truth boxes from bird's eye view. This performance of this metric is critical for autonomous driving to avoid collision. $A P_{3 D}$ counts the intersection of two cuboids (i.e., predicted box and ground truth) and adds object height and up-down information based on location.

Bird's Eye View Evaluation. $A P_{B E V}$ evaluates the projection of the 3D box on bird's eye view. For comprehensive comparison, we experiment with two IoU thresholds ( 0.5 and 0.7 ) following exsting methods. Just as shown in Tab. 1, our method outperforms state-of-the-art monocular based methods at both IoUs and surpasses Pseudo-LiDAR (Wang et al. 2019) by $4.91 \%$ for 0.5 IoU and $3.39 \%$ for 0.7 IoU under moderate level respectively.

3D Detection Evaluation. For $A P_{3 D}$, we also perform evaluations under the two IoUs. Compared to $A P_{B E V}$, $A P_{3 D}$ expands from bird's eye view plane to $3 \mathrm{D}$ space and calculates the intersection over union with $3 \mathrm{D}$ ground-truth boxes. As show in Tab. 1, our method outperforms stateof-the-art monocular methods in all difficulties for $0.5 \mathrm{IoU}$ and surpasses Pseudo-LiDAR (Wang et al. 2019) by $8.20 \%$ for moderate level. The results of 0.7 IoU have certain gap compared with Pseudo-LiDAR.

Results on Test Set. We submit our results to KITTI test server for evaluation and compare with all monocular based published methods on the test set. As shown in Tab. 2, the results show that our method outperforms the previous methods by significant margins in almost all metrics, which prove the effectiveness of our method. Compared to the latest state-of-the-art method, our $A P_{B E V}$ has an average improvement of $3.31 \%$, and $A P_{3 D}$ increases by $\sim 1 \%$ at easy level.

\section{Ablation Study}

In this section, we conduct ablation experiments to validate the effectiveness of different components of our overall framework. All comparison are engaged on validation set.

Benefits of Decoupled Tasks. As introduced in Decoupled Tasks, we decompose the 3D box estimation problem into sub-tasks including image based structured polygon estimation and height-guided depth estimation. To better evaluate the contribution of decoupled tasks, we compare our coarse and final results with regressing all variables simultaneously. As shown in Tab. 3, the performance of jointly regressing all parameters is far worse than our decoupled strategy. Even compared with the coarse results, there is a drop of $10 \%$ in terms of $A P_{B E V}$ and $A P_{3 D}$.

Benefits of Structured Polygon. We add an experiment of utilizing bird's eye view (BEV) features to regress coarse 3D boxes instead of using structured polygon (SP). As shown in Tab. 4, the model with structured polygon outperforms the one with BEV by $15.70 \%$ and $22.62 \%$ in terms of $A P_{B E V}$ and $A P_{3 D}$ at moderate level, which demonstrates the contribution of structured polygon. 
Table 1: Bird's eye view localization and 3D detection performance: Average Precision in bird's eye view $\left(A P_{B E V}\right)$ and Average Precision of $3 \mathrm{D}$ boxes $\left(A P_{3 D}\right)$ on KITTI validation set.

\begin{tabular}{|c||c|c|c|c|c|c|c|c|c|c|c|c|}
\hline \multirow{2}{*}{ Method } & \multicolumn{2}{|c|}{ IoU=0.5 $A P_{B E V}$} & \multicolumn{3}{c|}{ IoU=0.5 $A P_{3 D}$} & \multicolumn{3}{c|}{ IoU=0.7 $A P_{B E V}$} & \multicolumn{3}{c|}{ IoU=0.7 $A P_{3 D}$} \\
\cline { 2 - 12 } & Easy & Mod. & Hard & Easy & Mod. & Hard & Easy & Mod. & Hard & Easy & Mod. & Hard \\
\hline Mono3D & 30.50 & 22.93 & 19.16 & 25.19 & 18.20 & 15.52 & 5.22 & 5.19 & 4.13 & 2.53 & 2.31 & 2.31 \\
Deep3Dbox & 31.12 & 22.53 & 18.12 & 26.15 & 19.42 & 14.62 & 9.33 & 6.71 & 5.11 & 5.49 & 3.96 & 2.92 \\
MLF & 55.02 & 36.73 & 31.27 & 47.88 & 29.48 & 26.42 & 22.03 & 13.63 & 11.60 & 10.53 & 5.69 & 5.39 \\
ROI-10D & - & - & - & - & - & - & 14.76 & 9.55 & 7.57 & 10.25 & 6.39 & 6.18 \\
GS3D & - & - & - & 33.11 & 27.16 & 23.57 & - & - & - & 11.63 & 10.51 & 10.51 \\
MGR & 53.29 & 37.55 & 30.46 & 50.51 & 36.97 & 30.82 & 21.67 & 14.93 & 12.34 & 13.88 & 10.19 & 7.62 \\
MonoPSR & 56.97 & 43.39 & 36.00 & 49.65 & 41.71 & 29.95 & 20.63 & 18.67 & 14.45 & 12.75 & 11.48 & 8.59 \\
Pseudo-LiDAR & 70.8 & 49.4 & 42.7 & 66.3 & 42.3 & 38.5 & 40.6 & 26.3 & 22.9 & $\mathbf{2 8 . 2}$ & 18.5 & $\mathbf{1 6 . 4}$ \\
\hline Ours & $\mathbf{7 3 . 2 2}$ & $\mathbf{5 4 . 3 1}$ & $\mathbf{4 5 . 9 7}$ & $\mathbf{6 9 . 4 0}$ & $\mathbf{5 0 . 5 0}$ & $\mathbf{4 2 . 4 6}$ & $\mathbf{4 4 . 4 2}$ & $\mathbf{2 9 . 6 9}$ & $\mathbf{2 4 . 6 0}$ & 26.95 & $\mathbf{1 8 . 6 8}$ & 15.82 \\
\hline
\end{tabular}

Table 2: Average precision for bird's eye view localization and 3D detection on KITTI test set.

\begin{tabular}{|c||l|l|l|l|l|l|}
\hline \multirow{2}{*}{ Method } & \multicolumn{3}{|c|}{$A P_{B E V}$} & \multicolumn{3}{c|}{$A P_{3 D}$} \\
\cline { 2 - 7 } & Easy & Mod. & Hard & Easy & Mod. & Hard \\
\hline ROI-10D & 9.78 & 4.91 & 3.74 & 2.02 & 4.32 & 1.46 \\
GS3D & 8.41 & 6.08 & 4.94 & 2.90 & 4.47 & 2.47 \\
MGR & 18.19 & 11.17 & 8.73 & 9.61 & 5.74 & 4.25 \\
MonoPSR & 18.33 & 12.58 & 9.91 & 10.76 & 7.25 & $\mathbf{5 . 8 5}$ \\
\hline Ours & $\mathbf{2 4 . 6 2}$ & $\mathbf{1 4 . 6 6}$ & $\mathbf{1 1 . 4 6}$ & $\mathbf{1 1 . 6 8}$ & $\mathbf{7 . 2 8}$ & 5.69 \\
\hline
\end{tabular}

Table 3: Ablation study of decoupled tasks. Jointly represents all variables regressed simultaneously.

\begin{tabular}{|c||c|l|l|l|l|l|}
\hline \multirow{2}{*}{ Method } & \multicolumn{3}{|c|}{ IoU=0.5 $A P_{B E V}$} & \multicolumn{3}{c|}{ IoU $=0.5 A P_{3 D}$} \\
\cline { 2 - 7 } & Easy & Mod. & Hard & Easy & Mod. & Hard \\
\hline Jointly & 16.44 & 12.05 & 9.90 & 6.84 & 4.50 & 4.13 \\
\hline Coarse & 26.42 & 20.91 & 17.93 & 19.67 & 16.36 & 13.87 \\
\hline Ours & $\mathbf{7 3 . 2 2}$ & $\mathbf{5 4 . 3 1}$ & $\mathbf{4 5 . 9 7}$ & $\mathbf{6 9 . 4 0}$ & $\mathbf{5 0 . 5 0}$ & $\mathbf{4 2 . 4 6}$ \\
\hline
\end{tabular}

Benefits of Height-Guided Depth Estimation. As introduced, we propose a simple, but effective height-guided depth recovery strategy. To verify the effectiveness, we regress the depth directly and use mean depth error to compare the two strategies. As shown in Tab. 5, the mean depth error of height-guided depth recovery strategy is $1.21 \mathrm{~m}$, while directly regressing depth is $2.41 \mathrm{~m}$ almost twice as much as the former. The depth inferred from height outperforms regressing directly, which is due to that the height-guided strategy tackles the problem by utilizing the stable physical prior object height.

Benefits of 3D Box Refinement. As mentioned in $3 D$ Box Refinement, we propose a tailored refinement scheme for 3D task and argue that bird's eye view map with direct 3D spatial information is much more suitable than 2D image-level features and front-view depth map for fine-grained refinement in the 3D space. For comprehensive comparison, we refine coarse boxes with 2D image features and front-view depth map respectively. As shown in Tab. 6 , the impacts of 2D image-level features (i.e., $+i m g$ ) and front-view depth map (i.e., $+f v$ ) are basically the same. The results of $3 \mathrm{D}$ detection are all better than our coarse results and $A P_{3 D}$ improves about $1.3 \%$ at hard level.
Table 4: Ablation study of structured polygon. BEV represents coarse 3D boxes regressed with bird's eye view map.

\begin{tabular}{|c||c|l|l|l|l|l|}
\hline \multirow{2}{*}{ Method } & \multicolumn{2}{|c|}{ IoU $=0.5 A P_{B E V}$} & \multicolumn{3}{c|}{ IoU $=0.5 A P_{3 D}$} \\
\cline { 2 - 7 } & Easy & Mod. & Hard & Easy & Mod. & Hard \\
\hline BEV & 56.19 & 38.61 & 31.94 & 40.03 & 27.88 & 22.70 \\
\hline SP & $\mathbf{7 3 . 2 2}$ & $\mathbf{5 4 . 3 1}$ & $\mathbf{4 5 . 9 7}$ & $\mathbf{6 9 . 4 0}$ & $\mathbf{5 0 . 5 0}$ & $\mathbf{4 2 . 4 6}$ \\
\hline
\end{tabular}

Table 5: Ablation study of height-guided depth estimation. The lower the mean depth error value, the better the results.

\begin{tabular}{|c|c|}
\hline Method & mean depth error \\
\hline regress directly & $2.41 \mathrm{~m}$ \\
height-guided & $\mathbf{1 . 2 1} \mathrm{m}$ \\
\hline
\end{tabular}

The performance of localization is basically unchanged. While with the help of fine-grained $3 \mathrm{D}$ box refinement (i.e., $F G$ ), $A P_{B E V}$ and $A P_{3 D}$ have been substantially improved, which proves the effectiveness of $F G$ successfully capturing bird's eye view map.

Table 6: Ablation study of 3D box refinement. Comparison of different refinement strategies. $(+i m g)$ and $(+f v)$ represent refining the coarse results via image-level features and front-view depth map respectively.

\begin{tabular}{|c||c|c|c|c|c|c|}
\hline \multirow{2}{*}{ Method } & \multicolumn{3}{|c|}{ IoU $=0.5 A P_{B E V}$} & \multicolumn{3}{c|}{ IoU $=0.5 A P_{3 D}$} \\
\cline { 2 - 7 } & Easy & Mod. & Hard & Easy & Mod. & Hard \\
\hline Coarse & 26.42 & 20.91 & 17.93 & 19.67 & 16.36 & 13.87 \\
\hline$+i m g$ & 26.36 & 20.74 & 17.83 & 21.82 & 16.95 & 15.17 \\
$+f v$ & 26.32 & 20.70 & 17.80 & 21.81 & 16.93 & 15.14 \\
$+F G$ & $\mathbf{7 3 . 2 2}$ & $\mathbf{5 4 . 3 1}$ & $\mathbf{4 5 . 9 7}$ & $\mathbf{6 9 . 4 0}$ & $\mathbf{5 0 . 5 0}$ & $\mathbf{4 2 . 4 6}$ \\
\hline
\end{tabular}

The remarkable margins for our coarse to final results is due to two reasons. One is about the elaborately designed refinement scheme, which leverages bird's eye view map. Compared to other formats, our design with direct spatial information is much more suitable for $3 \mathrm{D}$ detection. Another is that most coarse boxes are actually not far from the corresponding targets. As shown in Fig. 8, most deviations of coarse $X$ and $Z$ are within $1 \mathrm{~m}$. As long as the boxes are shifted suitably, the accuracy can be increased substantially.

We also conduct an additional experiment to verify the contribution of FG. As shown Tab. 7, when applied on other 

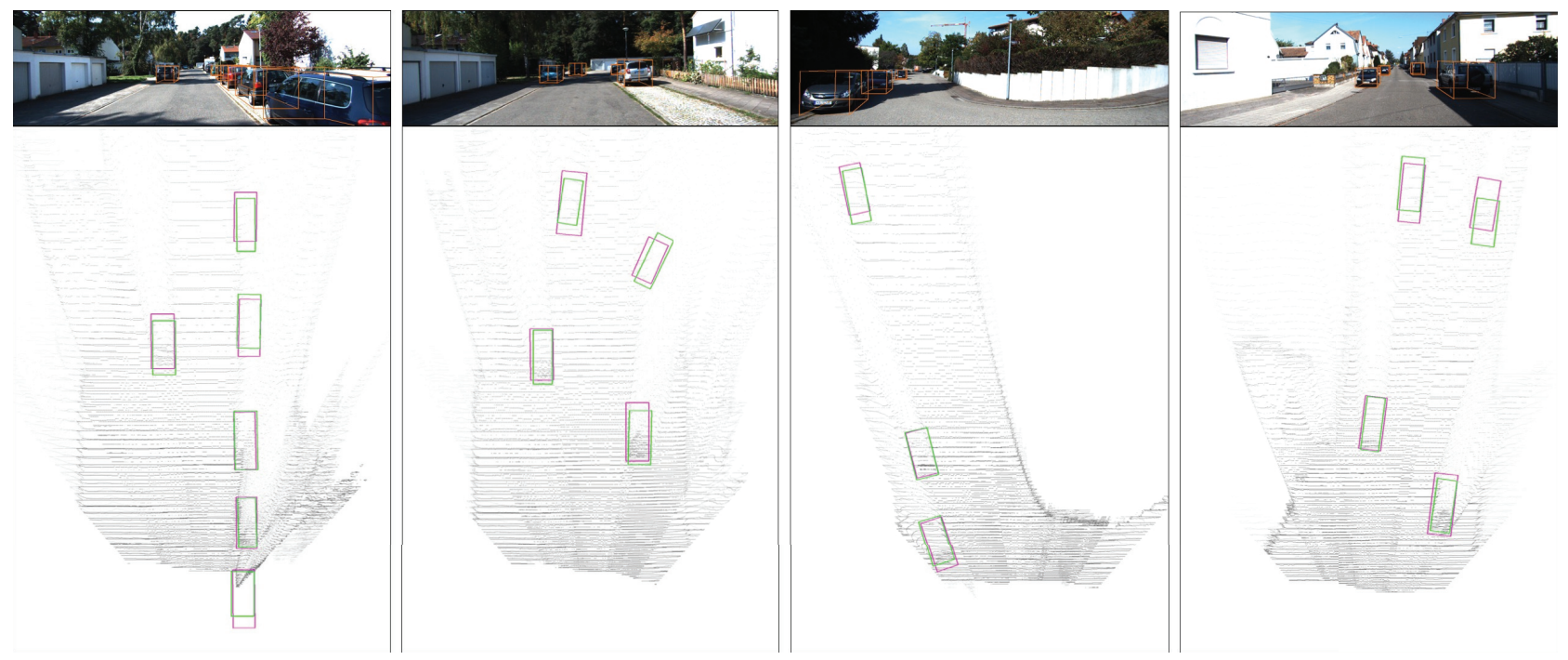

Figure 7: Qualitative Results. Top: structured polygons. Bottom: 3D bounding boxes in bird's eye view. Camera center is located at bottom center. Predicted 3D bounding boxes are drawn in pink, while ground truths are in green. Best viewed in color.

methods like Mono3D (Chen et al. 2016) and MGR (Qin, Wang, and Lu 2019), FG can also improve them with significant margins.

Table 7: Ablation study of 3D box refinement. Results for other methods refined similarly.

\begin{tabular}{|c||c|l|l|l|l|l|}
\hline \multirow{2}{*}{\multicolumn{1}{|c||}{ Method }} & \multicolumn{3}{c|}{ IoU $=0.5 A P_{B E V}$} & \multicolumn{3}{c|}{ IoU $=0.5 A P_{3 D}$} \\
\cline { 2 - 7 } & Easy & Mod. & Hard & Easy & Mod. & Hard \\
\hline Mono3D & 30.50 & 22.93 & 19.16 & 25.19 & 18.20 & 15.52 \\
Mono3D+FG & 54.12 & 37.67 & 32.20 & 41.04 & 29.05 & 24.59 \\
\hline MGR & 53.29 & 37.55 & 30.46 & 50.51 & 36.97 & 30.82 \\
MGR+FG & 65.87 & 49.01 & 40.93 & 60.95 & 43.80 & 36.27 \\
\hline
\end{tabular}

\section{Qualitative Results}

We show some quantitative results in Fig. 7, where structured polygons are visualized in top row and the 3D boxes are showed in bottom on bird's eye view. The pink and green boxes represent ground-truth boxes and predicted results respectively. As seen from the visualization results, our method can accurately predict the boxes in different locations and orientations only based on monocular images.

\section{Conclusion}

In this paper, we propose an efficient monocular 3D object detection framework which decomposes the complicated 3D object detection problem into a structured polygon prediction task and a following depth recovery task. The former task uses a stacked top-down/bottom-up hourglass network to build the structured polygon with a pretty high precision. The following depth recovery task utilizes the object height prior to inversely project the structured polygon to a cuboid in the 3D space. Moreover, a fine-grained refinement scheme is then adopted to rectify the deviations, which uses the local feature from the bird's eye view transformed from the

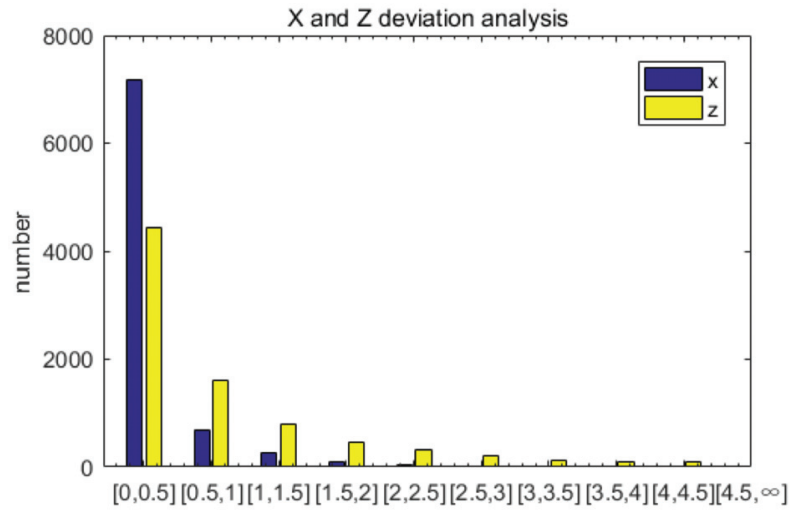

Figure 8: Deviation analysis of $X$ and $Z$. The figure shows the number of absolute deviation of $X$ and $Z$ of the coarse boxes compared with ground-truth boxes in different interval segments. Best viewed in color.

prediction of a monocular depth estimation algorithm. Experiments on the KITTI benchmark proves the effectiveness of our proposed framework.

\section{Acknowledgment}

This work is supported in part by SenseTime Group Limited, in part by the General Research Fund through the Research Grants Council of Hong Kong under Grants CUHK14202217, CUHK14203118, CUHK14207319.

\section{References}

Chabot, F.; Chaouch, M.; Rabarisoa, J.; Teuliere, C.; and Chateau, T. 2017. Deep manta: A coarse-to-fine many-task network for joint $2 \mathrm{~d}$ and $3 \mathrm{~d}$ vehicle analysis from monocular 
image. In Proceedings of the IEEE Conference on Computer Vision and Pattern Recognition, 2040-2049.

Chen, X.; Kundu, K.; Zhu, Y.; Berneshawi, A. G.; Ma, H.; Fidler, S.; and Urtasun, R. 2015. 3d object proposals for accurate object class detection. In Advances in Neural Information Processing Systems, 424-432.

Chen, X.; Kundu, K.; Zhang, Z.; Ma, H.; Fidler, S.; and Urtasun, R. 2016. Monocular 3d object detection for autonomous driving. In Proceedings of the IEEE Conference on Computer Vision and Pattern Recognition, 2147-2156.

Chen, X.; Ma, H.; Wan, J.; Li, B.; and Xia, T. 2017. Multiview $3 \mathrm{~d}$ object detection network for autonomous driving. In Proceedings of the IEEE Conference on Computer Vision and Pattern Recognition, 1907-1915.

Chen, X.; Kundu, K.; Zhu, Y.; Ma, H.; Fidler, S.; and Urtasun, R. 2018. 3d object proposals using stereo imagery for accurate object class detection. IEEE transactions on pattern analysis and machine intelligence 40(5):1259-1272.

Fu, H.; Gong, M.; Wang, C.; Batmanghelich, K.; and Tao, D. 2018. Deep Ordinal Regression Network for Monocular Depth Estimation. In IEEE Conference on Computer Vision and Pattern Recognition (CVPR).

Geiger, A.; Lenz, P.; and Urtasun, R. 2012. Are we ready for autonomous driving? the kitti vision benchmark suite. In Conference on Computer Vision and Pattern Recognition (CVPR).

Girshick, R. 2015. Fast r-cnn. In Proceedings of the IEEE international conference on computer vision, 1440-1448.

He, K.; Zhang, X.; Ren, S.; and Sun, J. 2015. Deep residual learning for image recognition. arXiv preprint arXiv:1512.03385.

Kehl, W.; Manhardt, F.; Tombari, F.; Ilic, S.; and Navab, N. 2017. Ssd-6d: Making rgb-based 3d detection and 6d pose estimation great again. In Proceedings of the IEEE International Conference on Computer Vision, 1521-1529.

Ku, J.; Pon, A. D.; and Waslander, S. L. 2019. Monocular $3 \mathrm{~d}$ object detection leveraging accurate proposals and shape reconstruction. In Proceedings of the IEEE Conference on Computer Vision and Pattern Recognition, 11867-11876.

Li, B.; Ouyang, W.; Sheng, L.; Zeng, X.; and Wang, X. 2019. Gs3d: An efficient 3d object detection framework for autonomous driving. In Proceedings of the IEEE Conference on Computer Vision and Pattern Recognition, 1019-1028.

Li, P.; Chen, X.; and Shen, S. 2019. Stereo r-cnn based $3 \mathrm{~d}$ object detection for autonomous driving. In Proceedings of the IEEE Conference on Computer Vision and Pattern Recognition, 7644-7652.

Luo, W.; Yang, B.; and Urtasun, R. 2018. Fast and furious: Real time end-to-end $3 \mathrm{~d}$ detection, tracking and motion forecasting with a single convolutional net. In Proceedings of the IEEE Conference on Computer Vision and Pattern Recognition, 3569-3577.

Manhardt, F.; Kehl, W.; and Gaidon, A. 2019. Roi-10d: Monocular lifting of $2 \mathrm{~d}$ detection to $6 \mathrm{~d}$ pose and metric shape. In Proceedings of the IEEE Conference on Computer Vision and Pattern Recognition, 2069-2078.
Mousavian, A.; Anguelov, D.; Flynn, J.; and Kosecka, J. 2017. 3d bounding box estimation using deep learning and geometry. In Proceedings of the IEEE Conference on Computer Vision and Pattern Recognition, 7074-7082.

Newell, A.; Yang, K.; and Deng, J. 2016. Stacked hourglass networks for human pose estimation. In European Conference on Computer Vision, 483-499. Springer.

Pepik, B.; Stark, M.; Gehler, P.; Ritschel, T.; and Schiele, B. 2015. 3d object class detection in the wild. In Proceedings of the IEEE Conference on Computer Vision and Pattern Recognition Workshops, 1-10.

Qi, C. R.; Su, H.; Mo, K.; and Guibas, L. J. 2017. Pointnet: Deep learning on point sets for $3 \mathrm{~d}$ classification and segmentation. In Proceedings of the IEEE Conference on Computer Vision and Pattern Recognition, 652-660.

Qi, C. R.; Liu, W.; Wu, C.; Su, H.; and Guibas, L. J. 2018. Frustum pointnets for $3 \mathrm{~d}$ object detection from rgb-d data. In Proceedings of the IEEE Conference on Computer Vision and Pattern Recognition, 918-927.

Qin, Z.; Wang, J.; and Lu, Y. 2019. Monogrnet: A geometric reasoning network for monocular $3 \mathrm{~d}$ object localization. In Proceedings of the AAAI Conference on Artificial Intelligence, volume 33, 8851-8858.

Ren, S.; He, K.; Girshick, R.; and Sun, J. 2015. Faster r-cnn: Towards real-time object detection with region proposal networks. In Advances in neural information processing systems, 91-99.

Russakovsky, O.; Deng, J.; Su, H.; Krause, J.; Satheesh, S.; Ma, S.; Huang, Z.; Karpathy, A.; Khosla, A.; Bernstein, M.; et al. 2015. Imagenet large scale visual recognition challenge. International journal of computer vision 115(3):211252.

Shi, S.; Wang, X.; and Li, H. 2019. Pointrenn: 3d object proposal generation and detection from point cloud. In Proceedings of the IEEE Conference on Computer Vision and Pattern Recognition, 770-779.

Wang, Y.; Chao, W.-L.; Garg, D.; Hariharan, B.; Campbell, M.; and Weinberger, K. Q. 2019. Pseudo-lidar from visual depth estimation: Bridging the gap in $3 \mathrm{~d}$ object detection for autonomous driving. In Proceedings of the IEEE Conference on Computer Vision and Pattern Recognition, 8445-8453.

Xu, B., and Chen, Z. 2018. Multi-level fusion based 3d object detection from monocular images. In Proceedings of the IEEE Conference on Computer Vision and Pattern Recognition, 2345-2353.

Zhou, Y., and Tuzel, O. 2018. Voxelnet: End-to-end learning for point cloud based $3 \mathrm{~d}$ object detection. In Proceedings of the IEEE Conference on Computer Vision and Pattern Recognition, 4490-4499. 